\title{
THE FUNDAMENTAL FORMULAS OF FINSLER SUBMANIFOLDS
}

\author{
JINTANG LI
}

\begin{abstract}
Let $\varphi:\left(M^{n}, F\right) \rightarrow\left(\bar{M}^{n+p}, \bar{F}\right)$ be an isometric immersion from a Finsler manifold to a Finsler manifold. In this paper, we shall obtain the Gauss and Codazzi equations with respect to the Chern connection on submanifolds $M$, by which we prove that if $M$ is a weakly totally geodesic submanifold of $\bar{M}$, then flag curvature of $M$ equals flag curvature of $\bar{M}$.
\end{abstract}

\section{Introduction}

Let $M$ be an $n$-dimensional smooth manifold and $\pi: T M \rightarrow M$ be the natural projection from the tangent bundle. Let $(x, Y)$ be a point of $T M$ with $x \in M, Y \in T_{x} M$ and let $\left(x^{i}, Y^{i}\right)$ be the local coordinates on $T M$ with $Y=Y^{i} \frac{\partial}{\partial x^{i}}$. A Finsler metric on $M$ is a function $F: T M \rightarrow[0,+\infty)$ satisfying the following properties:

(i) Regularity: $F(x, Y)$ is smooth in $T M \backslash 0$;

(ii) Positive homogeneity: $F(x, \lambda Y)=\lambda F(x, Y)$ for $\lambda>0$;

(iii) Strong convexity: The fundamental quadratic form

$$
g=g_{i j}(x, Y) d x^{i} \bigotimes d x^{j}
$$

is positively definite, where $g_{i j}=\frac{\partial^{2}\left[\frac{1}{2} F^{2}\right]}{\partial Y^{2} \partial Y^{j}}$.

It is well known that the Gauss, Codazzi and Ricci equations play an important role in studying the Riemannian submanifolds. To the author's knowledge, there is no one using the induced Chern connection in studying Finsler submanifolds. Firstly, in $\S 3$ we shall establish the Gauss and Codazzi equations of the Chern connection for Finsler submanifolds. In $\S 4$ we introduce the notion of weakly totally geodesic for Finsler submanifolds. By Gauss and Codazzi equations of the Chern connection we prove the following:

Theorem 4.4. If $M$ is a weakly totally geodesic submanifold of $\bar{M}$, then flag curvature of $M$ equals flag curvature of $\bar{M}$.

Received February 13, 2009; Revised March 16, 2009.

2000 Mathematics Subject Classification. 53C60, 53C40.

Key words and phrases. Finsler submanifolds, Gauss equation, weakly totally geodesic.

(c)2010 The Korean Mathematical Society 


\section{The Gauss formula}

Let $\left(M^{n}, F\right)$ be an $n$-dimensional Finsler manifold. $F$ inherits the Hilbert form and the Cartan tensor as follows:

$$
\omega=\frac{\partial F}{\partial Y^{i}} d x^{i}, A=A_{i j k} d x^{i} \otimes d x^{j} \otimes d x^{k}, \quad A_{i j k}:=F \frac{\partial g_{i j}}{\partial Y^{k}} .
$$

Let $\varphi:\left(M^{n}, F\right) \rightarrow\left(\bar{M}^{n+p}, \bar{F}\right)$ be an isometric immersion from a Finsler manifold to a Finsler manifold. We have [4]

$$
\begin{array}{r}
F(Y)=\bar{F}\left(\varphi_{*}(Y)\right), \quad g_{Y}(U, V)=\bar{g}_{\varphi_{*}(Y)}\left(\varphi_{*}(U), \varphi_{*}(V)\right), \\
A_{Y}(U, V, W)=\bar{A}_{\varphi_{*}(Y)}\left(\varphi_{*}(U), \varphi_{*}(V), \varphi_{*}(W)\right),
\end{array}
$$

where $Y, U, V, W \in T M, \bar{g}$ and $\bar{A}$ are the fundamental tensor and the Cartan tensor of $\bar{M}$, respectively.

It can be seen from (2.1) that

$$
\varphi^{*}(\bar{\omega})=\omega
$$

where $\bar{\omega}$ is the Hilbert form of $\bar{M}$.

We shall make use of the following convention:

$$
\varphi_{*}(U):=U, \quad \forall U \in T M,
$$

and

$$
1 \leq i, j, \ldots \leq n, \quad n+1 \leq \alpha, \beta, \ldots \leq n+p, \quad 1 \leq \lambda, \mu, \ldots \leq n-1 .
$$

Let $\varphi:\left(M^{n}, F\right) \rightarrow\left(\bar{M}^{n+p}, \bar{F}\right)$ be an isometric immersion from a Finsler manifold to a Finsler manifold. Take a $\bar{g}$-orthonormal frame form $\left\{e_{a}\right\}$ for $\pi^{*} T \bar{M}$ and $\left\{\omega^{a}\right\}$ be a local dual coframe such that $\left\{e_{i}\right\}$ is a frame field of $\pi^{*} T M$ and $\omega^{n}$ is the Hilbert form. Let $\theta_{b}^{a}$ and $\omega_{j}^{i}$ denote the Chern connection 1-form of $\bar{F}$ and $F$, respectively, i.e., $\bar{\nabla} e_{a}=\theta_{a}^{b} e_{b}$ and $\nabla e_{i}=\omega_{i}^{j} e_{j}$, where $\bar{\nabla}$ and $\nabla$ are the Chern connection of $\bar{M}$ and $M$, respectively. We obtain

$$
A\left(e_{i}, e_{j}, e_{n}\right)=0, \quad \bar{A}\left(e_{a}, e_{b}, e_{n}\right)=0, \quad \forall i, j, a, b .
$$

The structure equations of $\bar{M}$ are given by

$$
\left\{\begin{array}{l}
d \omega^{a}=-\theta_{b}^{a} \wedge \omega^{b}, \\
d \theta_{b}^{a}=-\theta_{c}^{a} \wedge \theta_{b}^{c}+\frac{1}{2} \bar{R}_{b c d}^{a} \omega^{c} \wedge \omega^{d}+\bar{P}_{b c d}^{a} \omega^{c} \wedge \theta_{n}^{d}, \\
\theta_{b}^{a}+\theta_{a}^{b}=-2 \bar{A}_{a b c} \theta_{n}^{c}, \\
\theta_{n}^{a}+\theta_{a}^{n}=0, \quad \theta_{n}^{n}=0 .
\end{array}\right.
$$

We have the following:

Theorem 2.1 (The Gauss formula). Let $\varphi:\left(M^{n}, F\right) \rightarrow\left(\bar{M}^{n+p}, \bar{F}\right)$ be an isometric immersion from a Finsler manifold to a Finsler manifold and $\bar{\nabla}$ be 
the Chern connection of $\bar{M}$. If

$$
\begin{aligned}
\bar{\nabla}_{U} V= & \nabla_{U} V+B(U, V)+\sum_{i}\left\{\bar{A}\left(U, V, \bar{\nabla}_{e_{i}} e_{n}-\nabla_{e_{i}} e_{n}\right)\right. \\
& \left.-\bar{A}\left(V, e_{i}, \bar{\nabla}_{U} e_{n}-\nabla_{U} e_{n}\right)-\bar{A}\left(U, e_{i}, \bar{\nabla}_{V} e_{n}-\nabla_{V} e_{n}\right)\right\} e_{i},
\end{aligned}
$$

where $U=u^{i} e_{i}, V=v^{i} e_{i} \in \Gamma\left(\pi^{*} T M\right)$ and $B(U, V)=u^{i} v^{j} h_{i j}^{\alpha} e_{\alpha} \in \Gamma\left(\pi^{*} T M\right)^{\perp}$, then $\nabla$ is the Chern connection of $M$.

Proof. (1). (Affine connection)

For $\forall U, V \in \Gamma\left(\pi^{*} T M\right)$ and $\forall f, h \in C^{\infty}\left(\pi^{*} T M\right)$, by $\bar{\nabla}$ is the Chern connection of $\bar{M}$, we have

$$
\begin{aligned}
\bar{\nabla}_{f U}(h V)= & f(U h) V+f h \nabla_{U} V+f h B(U, V)+f h \sum_{i}\left\{\bar{A}\left(U, V, \bar{\nabla}_{e_{i}} e_{n}-\nabla_{e_{i}} e_{n}\right)\right. \\
& \left.-\bar{A}\left(V, e_{i}, \bar{\nabla}_{U} e_{n}-\nabla_{U} e_{n}\right)-\bar{A}\left(U, e_{i}, \bar{\nabla}_{V} e_{n}-\nabla_{V} e_{n}\right)\right\} e_{i} .
\end{aligned}
$$

On the other hand, we also have

$$
\begin{aligned}
\bar{\nabla}_{f U}(h V)= & \nabla_{f U}(h V)+B(f U, h V)+\sum_{i}\left\{\bar{A}\left(f U, h V, \bar{\nabla}_{e_{i}} e_{n}-\nabla_{e_{i}} e_{n}\right)\right. \\
& \left.-\bar{A}\left(h V, e_{i}, \bar{\nabla}_{f U} e_{n}-\nabla_{f U} e_{n}\right)-\bar{A}\left(f U, e_{i}, \bar{\nabla}_{h V} e_{n}-\nabla_{h V} e_{n}\right)\right\} e_{i} .
\end{aligned}
$$

From (2.7), (2.8), we take

$$
B(f U, h V)=f h B(U, V) .
$$

It can be seen from (2.4) and (2.6) that

$$
\bar{\nabla}_{W} e_{n}-\nabla_{W} e_{n}=B\left(W, e_{n}\right)-\sum_{\lambda} \bar{A}\left(W, e_{\lambda}, B\left(e_{n}, e_{n}\right)\right) e_{\lambda},
$$

then

$$
\begin{aligned}
& \bar{A}\left(U, V, \bar{\nabla}_{W} e_{n}-\nabla_{W} e_{n}\right) \\
= & \bar{A}\left(U, V, B\left(W, e_{n}\right)\right)-\sum_{\lambda} \bar{A}\left(U, V, e_{\lambda}\right) \bar{A}\left(W, e_{\lambda}, B\left(e_{n}, e_{n}\right)\right) .
\end{aligned}
$$

So (2.6) is equivalent to

$$
\begin{aligned}
\bar{\nabla}_{U} V= & \nabla_{U} V+B(U, V)+\sum_{i}\left\{\bar{A}\left(U, V, B\left(e_{i}, e_{n}\right)\right)-\bar{A}\left(U, e_{i}, B\left(V, e_{n}\right)\right)\right. \\
& -\bar{A}\left(V, e_{i}, B\left(U, e_{i}\right)\right)-\sum_{\lambda} \bar{A}\left(U, V, e_{\lambda}\right) \bar{A}\left(e_{\lambda}, e_{i}, B\left(e_{n}, e_{n}\right)\right) \\
& +\sum_{\lambda} \bar{A}\left(U, e_{i}, e_{\lambda}\right) \bar{A}\left(e_{\lambda}, V, B\left(e_{n}, e_{n}\right)\right) \\
& \left.+\sum_{\lambda} \bar{A}\left(V, e_{i}, e_{\lambda}\right) \bar{A}\left(e_{\lambda}, U, B\left(e_{n}, e_{n}\right)\right)\right\} e_{i}
\end{aligned}
$$


and (2.7) is equivalent to

$(2.13)$

$$
\begin{aligned}
\bar{\nabla}_{f U} h V= & f(U h) V+f h \nabla_{U} V+f h B(U, V) \\
& +f h \sum_{i}\left\{\bar{A}\left(U, V, B\left(e_{i}, e_{n}\right)\right)-\sum_{\lambda} \bar{A}\left(U, V, e_{\lambda}\right) \bar{A}\left(e_{\lambda}, e_{i}, B\left(e_{n}, e_{n}\right)\right)\right. \\
& -\bar{A}\left(U, e_{i}, B\left(V, e_{n}\right)\right)+\sum_{\lambda} \bar{A}\left(U, e_{i}, e_{\lambda}\right) \bar{A}\left(e_{\lambda}, V, B\left(e_{n}, e_{n}\right)\right) \\
& \left.-\bar{A}\left(V, e_{i}, B\left(U, e_{i}\right)\right)+\sum_{\lambda} \bar{A}\left(V, e_{i}, e_{\lambda}\right) \bar{A}\left(e_{\lambda}, U, B\left(e_{n}, e_{n}\right)\right)\right\} e_{i} .
\end{aligned}
$$

From (2.9) and (2.11), (2.8) can be written as

(2.14)

$$
\begin{aligned}
\bar{\nabla}_{f U} h V= & \nabla_{f U} h Y+f h B(U, V) \\
& +f h \sum_{i}\left\{\bar{A}\left(U, V, B\left(e_{i}, e_{n}\right)\right)-\sum_{\lambda} \bar{A}\left(U, V, e_{\lambda}\right) \bar{A}\left(e_{\lambda}, e_{i}, B\left(e_{n}, e_{n}\right)\right)\right. \\
& -\bar{A}\left(U, e_{i}, B\left(V, e_{n}\right)\right)+\sum_{\lambda} \bar{A}\left(U, e_{i}, e_{\lambda}\right) \bar{A}\left(e_{\lambda}, V, B\left(e_{n}, e_{n}\right)\right) \\
& \left.-\bar{A}\left(V, e_{i}, B\left(U, e_{i}\right)\right)+\sum_{\lambda} \bar{A}\left(V, e_{i}, e_{\lambda}\right) \bar{A}\left(e_{\lambda}, U, B\left(e_{n}, e_{n}\right)\right)\right\} e_{i} .
\end{aligned}
$$

It follows from (2.13) and (2.14) that

$$
\nabla_{f U} h V=f h \nabla_{U} V+f(U h) V .
$$

(2). (Torsion freeness)

By (2.6), we obtain

$$
\bar{\nabla}_{U} V-\bar{\nabla}_{V} U=\nabla_{U} V+B(U, V)-\nabla_{V} U-B(V, U) .
$$

On the other hand, since $\bar{\nabla}$ is the Chern connection of $\bar{M}$, we have

$$
\bar{\nabla}_{U} V-\bar{\nabla}_{V} U=[U, V]
$$

It follows from (2.16) and (2.17) that

$$
\nabla_{U} V-\nabla_{V} U=[U, V]
$$

and

$$
B(U, V)=B(V, U) .
$$

(3). (Almost $g$-compatibility) 
For $\forall U, V, W \in \Gamma\left(\pi^{*} T M\right)$

$$
\begin{aligned}
U\langle V, W\rangle_{g}= & \left\langle\bar{\nabla}_{U} V, W\right\rangle_{\bar{g}}+\left\langle V, \bar{\nabla}_{U} W\right\rangle_{\bar{g}}+2 \bar{A}\left(V, W, \bar{\nabla}_{U} e_{n}\right) \\
= & \left\langle\nabla_{U} V, W\right\rangle_{g}+\left\{\bar{A}\left(U, V, \bar{\nabla}_{W} e_{n}-\nabla_{W} e_{n}\right)\right. \\
& \left.-\bar{A}\left(V, W, \bar{\nabla}_{U} e_{n}-\nabla_{U} e_{n}\right)-\bar{A}\left(U, W, \bar{\nabla}_{V} e_{n}-\nabla_{V} e_{n}\right)\right\} \\
& +\left\langle V, \nabla_{U} W\right\rangle_{g}+\left\{\bar{A}\left(U, W, \bar{\nabla}_{V} e_{n}-\nabla_{V} e_{n}\right)\right. \\
& \left.-\bar{A}\left(W, V, \bar{\nabla}_{U} e_{n}-\nabla_{U} e_{n}\right)-\bar{A}\left(U, V \bar{\nabla}_{W} e_{n}-\nabla_{W} e_{n}\right)\right\} \\
& +2 \bar{A}\left(V, W, \bar{\nabla}_{U} e_{n}\right) \\
= & \left\langle\nabla_{U} V, W\right\rangle_{g}+\left\langle V, \nabla_{U} W\right\rangle_{g}+2 A\left(V, W, \nabla_{U} e_{n}\right) .
\end{aligned}
$$

Combining (1), (2) and (3) completes the proof of Theorem 2.1 .

For $\forall U, V \in T M$ and $\xi \in T^{\perp} M$, we have

$$
0=U\langle V, \xi\rangle_{\bar{g}}=\langle B(U, V), \xi\rangle+\left\langle V, \bar{\nabla}_{U} \xi\right\rangle+2 \bar{A}\left(V, \xi, e_{a}\right) \theta_{n}^{a}(U) .
$$

Let $B\left(e_{i}, e_{j}\right)=h_{i j}^{\alpha} e_{\alpha}$. It follows from (2.21) that

$$
\theta_{\alpha}^{j}\left(e_{i}\right)=-h_{i j}^{\alpha}-2 \bar{A}_{j \alpha a} \theta_{n}^{a}\left(e_{i}\right)
$$

By the almost $\bar{g}$-compatibility, we have

$$
\theta_{\alpha}^{j}+\theta_{j}^{\alpha}=-2 \bar{A}_{j \alpha a} \theta_{n}^{a} .
$$

On the other hand, since $\omega^{\alpha}=0$ and the structure equations of $\bar{M}$, we have

$$
\theta_{j}^{\alpha} \wedge \omega^{j}=0
$$

It follows from $(2.22),(2.23)$ and $(2.24)$ that

$$
\theta_{j}^{\alpha}=h_{i j}^{\alpha} \omega^{i}
$$

and

$$
\theta_{\alpha}^{j}=-h_{i j}^{\alpha} \omega^{i}-2 \bar{A}_{j \alpha a} \theta_{n}^{a}
$$

From (2.12), we get

$$
\theta_{i}^{j}=\omega_{i}^{j}+\Psi_{j i k} \omega^{k}
$$

where

$(2.28)$

$\Psi_{j i k}=h_{j n}^{\alpha} \bar{A}_{k i \alpha}-h_{k n}^{\alpha} \bar{A}_{j i \alpha}-h_{i n}^{\alpha} \bar{A}_{k j \alpha}-h_{n n}^{\alpha} \bar{A}_{i k l} \bar{A}_{l j \alpha}+h_{n n}^{\alpha} \bar{A}_{i j l} \bar{A}_{l k \alpha}+h_{n n}^{\alpha} \bar{A}_{j k l} \bar{A}_{l i \alpha}$.

In particular,

$$
\theta_{n}^{j}=\omega_{n}^{j}-h_{n n}^{\alpha} \bar{A}_{j \lambda \alpha} \omega^{\lambda}, \quad \theta_{j}^{n}=\omega_{j}^{n}+h_{n n}^{\alpha} \bar{A}_{j \lambda \alpha} \omega^{\lambda} .
$$

It can be seen from (2.26) and (2.29) that

$$
\theta_{\alpha}^{j}=\left(-h_{i j}^{\alpha}-2 h_{n i}^{\beta} \bar{A}_{j \alpha \beta}+2 h_{n n}^{\beta} \bar{A}_{j \lambda \alpha} \bar{A}_{i \lambda \beta}\right) \omega^{i}-2 \bar{A}_{j \alpha \lambda} \omega_{n}^{\lambda} .
$$


In particular,

$$
\theta_{\alpha}^{n}=-h_{n i}^{\alpha} \omega^{i}
$$

\section{The Gauss equations}

By $(2.27) \sim(2.31)$ and the structure equations, we have

$$
\begin{aligned}
d \theta_{i}^{j}= & \theta_{i}^{k} \wedge \theta_{k}^{j}+\theta_{i}^{\alpha} \wedge \theta_{\alpha}^{j}+\frac{1}{2} \bar{R}_{i k l}^{j} \omega^{k} \wedge \omega^{l}+\bar{P}_{i k \lambda}^{j} \omega^{k} \wedge \theta_{n}^{\lambda}+\bar{P}_{i k \alpha}^{j} \omega^{k} \wedge \theta_{n}^{\alpha} \\
= & \omega_{i}^{k} \wedge \omega_{k}^{j}+\Psi_{k i l} \omega^{l} \wedge \omega_{k}^{j}+\Psi_{j k l} \omega_{i}^{k} \wedge \omega^{l}+\Psi_{k i s} \Psi_{j k l} \omega^{s} \wedge \omega^{l} \\
& -h_{i k}^{\alpha} h_{j l}^{\alpha} \omega^{k} \wedge \omega^{l}-2 h_{i k}^{\alpha} h_{n l}^{\beta} \bar{A}_{j \alpha \beta} \omega^{k} \wedge \omega^{l}+2 h_{i k}^{\alpha} h_{n n}^{\beta} \bar{A}_{j s \alpha} \bar{A}_{l s \beta} \omega^{k} \wedge \omega^{l} \\
& -2 h_{i k}^{\alpha} \bar{A}_{j \alpha \lambda} \omega^{k} \wedge \omega_{n}^{\lambda}+\frac{1}{2} \bar{R}_{i k l}^{j} \omega^{k} \wedge \omega^{l} \\
& +\bar{P}_{i k \lambda}^{j} \omega^{k} \wedge \omega_{n}^{\lambda}-h_{n n}^{\alpha} \bar{A}_{l s \alpha} \bar{P}_{i k s}^{j} \omega^{k} \wedge \omega^{l}+h_{n l}^{\alpha} \bar{P}_{i k \alpha}^{j} \omega^{k} \wedge \omega^{l} \\
= & \omega_{i}^{k} \wedge \omega_{k}^{j}++\Psi_{k i l} \omega^{l} \wedge \omega_{k}^{j}+\Psi_{j k l} \omega_{i}^{k} \wedge \omega^{l} \\
& +\left\{\Psi_{s i k} \Psi_{j s l}-h_{i k}^{\alpha} h_{j l}^{\alpha}-2 h_{i k}^{\alpha} h_{n l}^{\beta} \bar{A}_{j \alpha \beta}+2 h_{i k}^{\alpha} h_{n n}^{\beta} \bar{A}_{j s \alpha} \bar{A}_{l s \beta}\right. \\
& \left.+\frac{1}{2} \bar{R}_{i k l}^{j}-h_{n n}^{\alpha} \bar{A}_{l s \alpha} \bar{P}_{i k s}^{j}+h_{n l}^{\alpha} \bar{P}_{i k \alpha}^{j}\right\} \omega^{k} \wedge \omega^{l} \\
& +\left\{-2 h_{i k}^{\alpha} \bar{A}_{j \alpha \lambda}+\bar{P}_{i k \lambda}^{j}\right\} \omega^{k} \wedge \omega_{n}^{\lambda} .
\end{aligned}
$$

On the other hand, we also have

$$
d \omega_{i}^{j}=\omega_{i}^{k} \wedge \omega_{k}^{j}+\frac{1}{2} R_{i k l}^{j} \omega^{k} \wedge \omega^{l}+P_{i k \lambda}^{j} \omega^{k} \wedge \omega_{n}^{\lambda}
$$

and

$$
\begin{aligned}
d\left(\Psi_{j i k} \omega^{k}\right)= & \Psi_{j i k \mid l} \omega^{l} \wedge \omega^{k}+\Psi_{j i k ; \lambda} \omega_{n}^{\lambda} \wedge \omega^{k}+\Psi_{l i k} \omega_{j}^{l} \wedge \omega^{k}+\Psi_{j l k} \omega_{i}^{l} \wedge \omega^{k} \\
= & \Psi_{k i l} \omega^{l} \wedge \omega_{k}^{j}+\Psi_{j k l} \omega_{i}^{k} \wedge \omega^{l} \\
& +\Psi_{j i k \mid l} \omega^{l} \wedge \omega^{k}+\left\{-\Psi_{j i k ; \lambda}+2 \Psi_{s i k} A_{j s \lambda}\right\} \omega^{k} \wedge \omega_{n}^{\lambda} .
\end{aligned}
$$

Substituting (3.1), (3.2) and (3.2) into (2.27) implies

$$
\begin{array}{r}
\left(-2 h_{i j}^{\alpha} \bar{A}_{j \lambda \alpha}+\bar{P}_{i k \lambda}^{j}\right) \omega^{k} \wedge \omega_{n}^{\lambda}+\left(\Psi_{s i k} \Psi_{j s l}-h_{i k}^{\alpha} h_{j l}^{\alpha}-2 h_{i k}^{\alpha} h_{n l}^{\beta} \bar{A}_{j \alpha \beta}\right. \\
\left.\quad+2 h_{i k}^{\alpha} h_{n n}^{\beta} \bar{A}_{j s \alpha} \bar{A}_{l s \beta}+\frac{1}{2} \bar{R}_{i k l}^{j}-h_{n n}^{\alpha} \bar{P}_{i k s}^{j} \bar{A}_{s l \alpha}+h_{n l}^{\alpha} \bar{P}_{i k \alpha}^{j}\right) \omega^{k} \wedge \omega^{l} \\
=\left(P_{i k \lambda}^{j}-\Psi_{j i k ; \lambda}+2 \Psi_{s i k} A_{j s \lambda}\right) \omega^{k} \wedge \omega_{n}^{\lambda}+\left(\frac{1}{2} R_{i k l}^{j}-\Psi_{j i k \mid l}\right) \omega^{k} \wedge \omega^{l} .
\end{array}
$$

Thus we have the following result:

Theorem 3.1 (The Gauss equations). Let $\varphi:\left(M^{n}, F\right) \rightarrow\left(\bar{M}^{n+p}, \bar{F}\right)$ be an isometric immersion from a Finsler manifold to a Finsler manifold. Then we 
have

$$
\left\{\begin{aligned}
P_{i k \lambda}^{j}= & \bar{P}_{i k \lambda}^{j}+\Psi_{j i k ; \lambda}-2 \Psi_{s i k} A_{j s \lambda}-2 h_{i k}^{\alpha} \bar{A}_{j \lambda \alpha}, \\
R_{i k l}^{j}= & \bar{R}_{i k l}^{j}-h_{i k}^{\alpha} h_{j l}^{\alpha}+h_{i l}^{\alpha} h_{j k}^{\alpha}+\Psi_{j i k \mid l}-\Psi_{j i l \mid k} \\
& +\Psi_{s i k} \Psi_{j s l}-\Psi_{s i l} \Psi_{k s l}-2 h_{i k}^{\alpha} h_{n l}^{\beta} \bar{A}_{j \alpha \beta}+2 h_{i l}^{\alpha} h_{n k}^{\beta} \bar{A}_{j \alpha \beta} \\
& 2 h_{i k}^{\alpha} h_{n n}^{\beta} \bar{A}_{j s \alpha} \bar{A}_{l s \beta}-2 h_{i l}^{\alpha} h_{n n}^{\beta} \bar{A}_{j s \alpha} \bar{A}_{k s \beta}-h_{n n}^{\alpha} \bar{A}_{s l \alpha} \bar{P}_{i k s} \\
& +h_{n n}^{\alpha} \bar{A}_{s k \alpha} \bar{P}_{i l s}^{j}+h_{n l}^{\alpha} \bar{P}_{i k \alpha}^{j}-h_{n k}^{\alpha} \bar{P}_{i l \alpha}^{j} .
\end{aligned}\right.
$$

Exterior differentiate the left hand side term of (2.25), we obtain

$$
\begin{aligned}
d \theta_{i}^{\alpha}= & \theta_{i}^{k} \wedge \theta_{k}^{\alpha}+\theta_{i}^{\beta} \wedge \theta_{\beta}^{\alpha}+\frac{1}{2} \bar{R}_{i k l}^{\alpha} \omega^{k} \wedge \omega^{l}+\bar{P}_{i k \lambda}^{\alpha} \omega^{k} \wedge \theta_{n}^{\lambda}+\bar{P}_{i k \beta}^{\alpha} \omega^{k} \wedge \theta_{n}^{\beta} \\
= & h_{k j}^{\alpha} \omega_{i}^{k} \wedge \omega^{j}+h_{k j}^{\alpha} \Psi_{k i l} \omega^{l} \wedge \omega^{j}+h_{i k}^{\beta} \omega^{k} \wedge \theta_{\beta}^{\alpha}+\frac{1}{2} \bar{R}_{i k l}^{\alpha} \omega^{k} \wedge \omega^{l} \\
& +\bar{P}_{i k \lambda}^{\alpha} \omega^{k} \wedge \omega_{n}^{\lambda}-h_{n n}^{\beta} \bar{A}_{l s \beta} \bar{P}_{i k l}^{\alpha} \omega^{k} \wedge \omega^{s}+h_{n l}^{\beta} \bar{P}_{i k \beta}^{\alpha} \omega^{k} \wedge \omega^{l} \\
= & \left\{h_{s l}^{\alpha} \Psi_{s i k}+\frac{1}{2} \bar{R}_{i k l}^{\alpha}-h_{n n}^{\beta} \bar{A}_{s l \beta} \bar{P}_{i k s}+h_{n l}^{\beta} \bar{P}_{i k \beta}^{\alpha}\right\} \omega^{k} \wedge \omega^{l} \\
& +\bar{P}_{i k \lambda}^{\alpha} \omega^{k} \wedge \omega_{n}^{\lambda}+h_{k j}^{\alpha} \omega_{i}^{k} \wedge \omega^{j}+h_{i k}^{\beta} \omega^{k} \wedge \theta_{\beta}^{\alpha} .
\end{aligned}
$$

Exterior differentiate the right hand side term of (2.25), we also obtain

$$
\begin{aligned}
d\left(h_{i j}^{\alpha} \omega^{j}\right) & =h_{i j \mid k}^{\alpha} \omega^{k} \wedge \omega^{j}+h_{i j ; \lambda}^{\alpha} \omega_{n}^{\lambda} \wedge \omega^{j}+h_{k j}^{\alpha} \omega_{i}^{k} \wedge \omega^{j}-h_{i j}^{\beta} \theta_{\beta}^{\alpha} \wedge \omega^{j} \\
& =h_{i l \mid k}^{\alpha} \omega^{k} \wedge \omega^{l}-h_{i k ; \lambda}^{\alpha} \omega^{k} \wedge \omega_{n}^{\lambda}+h_{k j}^{\alpha} \omega_{i}^{k} \wedge \omega^{j}+h_{i k}^{\beta} \omega^{k} \wedge \theta_{\beta}^{\alpha} .
\end{aligned}
$$

It follows from (3.6) and (3.7) that

$$
\begin{aligned}
& {\left[\bar{P}_{i k \lambda}^{\alpha}+h_{i k ; \lambda}^{\alpha}\right] \omega^{k} \wedge \omega_{n}^{\lambda}} \\
& +\left[\frac{1}{2} \bar{R}_{i k j}^{\alpha}-h_{i j \mid k}^{\alpha}+h_{n j}^{\beta} \bar{P}_{i k \beta}^{\alpha}-h_{l k}^{\alpha} \Psi_{l i j}-h_{n n}^{\beta} \bar{A}_{l j \beta} \bar{P}_{i k l}^{\alpha}\right] \omega^{k} \wedge \omega^{j}=0 .
\end{aligned}
$$

From (3.8), we may state the following theorem:

Theorem 3.2 (The Codazzi equations). Let $\varphi:\left(M^{n}, F\right) \rightarrow\left(\bar{M}^{n+p}, \bar{F}\right)$ be an isometric immersion from a Finsler manifold to a Finsler manifold. Then we have

$$
\left\{\begin{aligned}
h_{i j ; \lambda}^{\alpha}= & -\bar{P}_{i j \lambda}^{\alpha} \\
h_{i j \mid k}^{\alpha}-h_{i k \mid j}^{\alpha}= & -\bar{R}_{i j k}^{\alpha}+h_{n j}^{\beta} \bar{P}_{i k \beta}^{\alpha}-h_{n k}^{\beta} \bar{P}_{i j \beta}^{\alpha} \\
& -h_{l k}^{\alpha} \Psi_{l i j}+h_{l j}^{\alpha} \Psi_{l i k}-h_{n n}^{\beta} \bar{A}_{l j \beta} \bar{P}_{i k l}^{\alpha}+h_{n n}^{\beta} \bar{A}_{l k \beta} \bar{P}_{i j l}^{\alpha}
\end{aligned}\right.
$$

\section{Some application of the fundamental formulas}

In this section, we shall use the fundamental formulas of the Finsler submanifolds to prove some results.

Definition 4.1. Let $\varphi:(M, F) \rightarrow(\bar{M}, \bar{F})$ be an isometric immersion. $B$ defined by (2.6) is called the second fundamental form of $\varphi . H=\frac{1}{n} \operatorname{tr} B=$ $\frac{1}{n} \sum_{i} B\left(e_{i}, e_{i}\right)=\frac{1}{n} \sum_{i, \alpha} h_{i i}^{\alpha} e_{\alpha}$ is called the mean curvature vector field of $\varphi . M$ is called to be minimal (or totally geodesic) if $H$ (or $B$ ) vanishes identically. 
When $M$ is a geodesic curve of $\bar{M}$, we have $\bar{\nabla}_{e_{1}} e_{1}=0$, i.e., $B\left(e_{1}, e_{1}\right)=0$, this implies the following:

Theorem 4.2. A geodesic curve is totally geodesic.

Definition 4.3. A submanifold $(M, F)$ of $(\bar{M}, \bar{F})$ is said to be weakly totally geodesic if $B\left(e_{n}, e_{n}\right)=0$.

Remark. We find it hard to explain the difference between "weakly totally geodesic" and "totally geodesic" by examples.

Theorem 4.4. If $M$ is a weakly totally geodesic submanifold of $\bar{M}$, then flag curvature of $M$ equals flag curvature of $\bar{M}$.

Proof. Since $M$ is weakly totally geodesic, we have

$$
h_{n n}^{\alpha}=0, \forall \alpha .
$$

Thus we get

$$
\begin{aligned}
h_{n n \mid i}^{\alpha} \omega^{i}+h_{n n ; \lambda}^{\alpha} \omega_{n}^{\lambda} & =d h_{n n}^{\alpha}-2 h_{\lambda n}^{\alpha} \omega_{n}^{\lambda}+h_{n n}^{\beta} \theta_{\beta}^{\alpha} \\
& =-2 h_{\lambda n}^{\alpha} \omega_{n}^{\lambda},
\end{aligned}
$$

from which we have

$$
\left\{\begin{array}{l}
h_{n n \mid i}^{\alpha}=0, \\
h_{n n ; \lambda}^{\alpha}=-2 h_{\lambda n}^{\alpha} .
\end{array}\right.
$$

On the other hand, using the fact $\bar{P}_{n n \lambda}^{\alpha}=0$ and the first formula of (3.9), we obtain

$$
h_{n n ; \lambda}^{\alpha}=0 \text {. }
$$

It follows from (4.4) and the second formula of (4.3) that

$$
h_{\lambda n}^{\alpha}=0, \forall \lambda, \alpha .
$$

Substituting (4.1) and (4.5) into (2.28) yields

$$
\Psi_{i j k}=0, \forall i, j, k \text {. }
$$

Substituting (4.1), (4.5) and (4.6) into the second formula of (3.5) implies

$$
R_{i k l}^{n}=\bar{R}_{i k l}^{n},
$$

which and the definition of flag curvature for the flag $\left(e_{n} \wedge V\right)$ yields

$$
K\left(e_{n}, V\right)=\frac{1}{|V|^{2}} R\left(e_{n}, V, e_{n}, V\right)=\frac{1}{|V|^{2}} \bar{R}\left(e_{n}, V, e_{n}, V\right)=\bar{K}\left(e_{n}, V\right),
$$

which completes the proof of Theorem 4.4.

Corollary. Let $(\bar{M}, \bar{F})$ be a Finsler manifold with constant flag curvature $c$. If $M$ is a weakly totally geodesic submanifold of $\bar{M}$, then $M$ has constant flag curvature $c$.

Acknowledgment. The author wishes to thank the referee for a careful reading of the manuscript and for very helpful suggestions. 


\section{References}

[1] D. Bao and S. S. Chern, On a notable connection in Finsler geometry, Houston J. Math. 19 (1993), no. 1, 135-180.

[2] D. Bao, S. S. Chern, and Z. Shen, An Introduction to Riemann-Finsler Geometry, Graduate Texts in Mathematics, 200. Springer-Verlag, New York, 2000.

[3] A. Bejancu, Structure equations for Riemann-Finsler subspaces, C. R. Acad. Bulgare Sci. 40 (1987), no. 1, 37-40.

[4] Z. Shen, On Finsler geometry of submanifolds, Math. Ann. 311 (1998), no. 3, 549-576.

Department of Mathematics

XiAMEN UNIVERSITY

361005 Xiamen, Fujian, P. R. China

E-mail address: dli66@xmu.edu.cn 